\title{
A TÉCNICA LIMPA DO AUTOCATETERISMO VESICAL INTERMITENTE: DESCRIÇÃO DO PROCEDIMENTTO REALIZADO PELOS PACIENTES COM LESÃO MEDULAR ${ }^{1}$
}

\author{
THE CLEAN TECHINIQUE OF VESICAL AUTOCATHETERISM INTTERMITENT: \\ DESCRIPTION OF THE PROCEDURE ACCOMPHISHED BY THE PACIENT \\ WITH MEDULLAR INJURY
}

\section{LA TECNICA LIMPIA DEL AUTOCATETERISMO VESICAL INTERMITENTE: DESCRIPCIÓN DEL PREOCDIMENTO REALIZADO POR LOS PACIENTES COM LESION MEDULAR}

\author{
Mitiko Moroóka* \\ Ana Cristina Mancussi e Faro**
}

\begin{abstract}
Moroóka M, Faro ACM. A técnica limpa do autocateterismo vesical intermitente: descrição do procedimento realizado pelos pacientes com lesão medular. Rev Esc Enferm USP 2002; 36(4): 324-31.

\section{RESUMO}

Neste trabalho, as autoras descrevem a seqüência da técnica e os materiais utilizados na realização do autocateterismo vesical intermitente - técnica limpa. As informações foram obtidas junto aos pacientes que realizam este procedimento $e$ que foram orientados no Serviço de Atendimento Interdisciplinar ao paciente com lesão medular traumática. Pretendemos, através da divulgação dos resultados, contribuir para a ampliação do conhecimento sobre o autocateterismo vesical e subsidiar a prática aos pacientes iniciantes neste processo terapêutico.
\end{abstract}

PALAVRAS-CHAVE: Reabilitação. Bexiga neurogênica. Medula. Enfermagem em reabilitação. ABSTRACT

The present study describes the sequence and its materials used in the vesical autocatheterism intermittent - clean technique. The information were obtained together with the patients, who go through such procedure and were directed to follow the instructions, given by the inter disciplinary Service, attending patients with traumatic medullar injury. The study intends to contribute to increase the knowledge about the vesical autocatheterism and provide to the patients this technique in a therapeutic way.

KEYWORDS: Rehabilitation. Neurogenic bladder. Bone marrow. Rehabilitation nursing.

\section{RESUMEN}

En este trabajo, las autoras describen la secuencia de la técnica y los materiales utilizados en la realización del autocateterismo vesical intermitente-ténica limpia. Las informaciones fueron orientadas em el Servicio de Atendimiento Interdisciplinar al paciente con lesión medular traumática. Pretendemos, através de la divulgaciín de los resultados, contribuir para la ampliación del conocimiento sobre el autocateterismo vesical y subvencionar la práctica a los pacientes iniciantes en este procedimento terapéutico, paciente.

PALABRAS-CLAVE: Reabilitación. Vejiga neurogénica. Medula óssea. Enfermería en reabilitación.

\footnotetext{
${ }^{1}$ Parte da Dissertação de Mestrado apresentada à Escola de Enfermagem da Universidade de São Paulo para obtenção do título de Mestre em Enfermagem.

* Docente Mestre do Departamento de Enfermagem da Universidade Estadual de Londrina.

** Livre Dqcente do Departamento de Enfermagem Médico-Cirúrgica da Escola de Enfermagem da USE
} 


\section{INTRODUÇÃO}

O traumatismo raquimedular é uma agressão física que inesperadamente anula ou limita o uso e controle do corpo, causando grande repercussão no indivíduo, na família e na sociedade.

E, uma vez que estruturas sociais são desestabilizadas, necessitam de uma reorganização e trocas de papéis exercidas até então.

Para os profissionais de saúde, assistir estes pacientes é considerado uma atribuição importante e gratificante, porém complexa, já que a reabilitação depende da orientação desta equipe.

$\mathrm{Na}$ fase inicial da lesão medular, a prevenção das complicações é de responsabilidade da enfermagem, sobretudo do enfermeiro, onde o cuidado fisico assume grande importância(1). A higiene e o conforto físico, mudança de decúbito e a reeducação vesical foram considerados pelos enfermeiros cuidados imprescindiveis na prevenção de sequelas físicas no paciente com lesão medular(2).

Compondo com o enfermeiro e compartilhando a responsabilidade pelo cuidado físico e motor estão outros profissionais de reabilitação como fisioterapeutas, médicos fisiatras, terapeuta ocupacional tornando a reabilitação do paciente precoce, minimizando complicações ${ }^{(3-4)}$.

As complicações digestivas, urinárias, deformidades, úlceras de pressão, trombose venosa profunda e disfunções autonômicas são citadas por Lianza(5) como intercorrências comuns na fase inicial da lesão medular. Outras disfunções, tais como hipotensão postural, ossificação para-articular, dor por desaferentação, espasticidade, recondicionamento vesical e intestinal são condições físicas que necessitam de intervenções do profissional de reabilitação.

O presente trabalho descreve dentro do enfoque de reabilitação e prevenção de complicações, o gerenciamento de bexiga neurogênica realizado por um grupo de pacientes com lesão medular.

$\mathrm{O}$ interesse pelo tema surgiu durante a nossa atuação como docente de Enfermagem em diferentes locais (ambulatórios, Unidades Básicas de Saúde, enfermarias) ao depararmos com pacientes que apresentam disfunção vesicoesfincteriana à procura de assistência para realizar a troca ou retirada de sonda de demora.

Faro(6) estudou os diagnósticos e intervenções de enfermagem voltados ao paciente com lesão medular em reabilitação. Como diagnóstico de enfermagem, esta autora encontrou o diagnóstico de incontinência urinária como um dos problemas mais freqüentes. Quanto às intervenções, vale destacar uma intervenção que retrata a necessidade de "treinar" o paciente e a família para a realização do autocateterismo vesical intermitente - técnica limpa.

Guttmann(7) em 1944 promovia o esvaziamento da bexiga, com intervalo pré-estabelecido, isto reduzia a infecção urinária. Nesta época por acreditar que a infecção urinária era causada pela introdução de microrganismo na bexiga através do cateter, preconizava o uso da técnica asséptica, o que exigia grande dedicação do pessoal médico, por isso não se adaptou ao uso ambulatorial.

Em 1972, Lapides(8) trouxeram grande contribuição quando introduziram o autocateterismo intermitente - técnica limpa, ressaltando que a manutenção da bexiga em estado fisiológico normal e condições de defesa do hospedeiro eram suficientes para prevenir a infecção. Após quatro anos, através de outras pesquisas concluíram que o autocateterismo intermitente diminui a infecção urinária e em alguns casos promovem retorno da função vesical.

o autocateterismo vesical intermitente permite alguma distensão da bexiga, o que representa o estímulo fisiológico para a micção e emite impulsos apropriados para o núcleo espinhal de controle vesical, promovendo desta forma o retorno da atividade do músculo detrusor (9-11)

A técnica limpa do autocateterismo vesical intermitente é um recurso seguro para, os pacientes com disfunção vesicoesfincteriana, já comprovado por pesquisas nacionais e internacionais $(3,6,8,12)$ É um procedimento considerado de fácil execução, que mais se aproxima da função vesical normal, reduz episódios de infecção urinária, melhora a auto-estima e preserva a função renal. Outras vantagens são: relação custo beneficio, promoção de reeducação vesical, favorecendo a miccção espontânea e tornando-se livre das cateterizações.

No entanto, constatamos no nosso cotidiano que existem muitas dúvidas na realização e orientação deste procedimento. Os questionamentos quanto aos materiais utilizados, beneficios/maleficios, prognósticos e complicações pairam entre as pessoas que realizam o autocateterismo em domicilio.

Assim, em face da complexidade das alterações, muitas vezes irreversíveis decorrentes da lesão medular, neste estudo, destacando a disfunção vesicoesfincteriana, descreveremos a vivência do procedimento do autocateterismo intermitente técnica limpa por um grupo de pessoas com lesão medular.

Temos, como finalidade maior, a ampliação do conhecimento do enfermeiro sobre o autocateterismo e subsidiar os pacientes iniciantes nesta prática que enquanto um tratamento, por excelência, visa oferecer melhores condições de vida social e de saúde. 


\section{OBJETIVO}

Descrever o procedimento do autocateterismo vesical intermitente - técnica limpa, realizado pelos pacientes com disfunção vesicoesfincteriana.

\section{MATERIAL E MÉTODO}

Este estudo foi do tipo exploratório e descritivo, realizado junto ao Serviço de Ambulatório do Hospital de Clínicas de Londrina e Hospital Universitário, que são órgãos suplementares da Universidade Estadual de Londrina.

Os docentes envolvidos com serviço de extensão, pesquisa e ensino, atendem pessoas com lesão medular de Londrina e outras regiões do Paraná há 13 anos.

A população foi composta por 22 pacientes que apresentam disfunção vesicoesfincteriana por lesão medular e que atenderam aos seguintes critérios de elegibilidade:

- homens e mulheres com lesão medular;

- com idade mínima de 18 anos;

- realizarem autocateterismo vesical intermitente técnica limpa há, no mínimo, um mês;

- serem pacientes cadastrados no projeto de atendimento interdisciplinar;

- serem moradores na região urbana do município de Londrina;

- concordarem por escrito em participar do estudo.

\section{Procedimento de coleta de dados:}

Os dados relacionados à descrição do procedimento foram coletados mediante entrevista realizada pela pesquisadora.

Foi solicitado ao paciente a leitura e posterior assinatura do termo de consentimento livre e esclarecido para participação no estudo.

Todos já tinham recebido treinamento sobre a técnica limpa do autocateterismo vesical intermitente e orientações referentes ao material necessário e local onde deve ser realizada.

\section{RESULTADOS E DISCUSSÃO}

Os resultados apresentados retratam a realização do procedimento autocateterismo vesical intermitente - técnica limpa, por pacientes com lesão medular, descrevendo-o passo a passo, esboçando em gráficos ou tabelas e comentando quando pertinentes.
Tabela 1 - Distribuição dos pacientes com lesão medular quanto à realização da higiene das mãos antes do autocateterismo vesical. Londrina-PR, 1999.

\begin{tabular}{lrc}
\hline \multicolumn{1}{c}{ higiene } & $\mathbf{n}$ & \% \\
\hline Sempre faz & 19 & 86,4 \\
Faz na maioria das vezes & 1 & 4,5 \\
Faz raramente & 1 & 4,5 \\
Nunca faz & 1 & 4,5 \\
\hline \multicolumn{1}{c}{ TOTAL } & $\mathbf{2 2}$ & $\mathbf{1 0 0 , 0}$ \\
\hline
\end{tabular}

Do total de pacientes, $19(86,4 \%)$ sempre fazem a higiene das mãos antes da realização do autocateterismo vesical.

Kawagoe(13), em normas básicas para a realização de procedimentos, ressaltam a importância da lavagem das mãos como a medida mais importante para prevenir a disseminação da infecção. As mãos devem ser lavadas, mesmo se foram utilizadas luvas, após tocar qualquer material contaminado, antes e após a realização de qualquer procedimento.

Froemming(14) ressaltam a importância da lavagem das mãos antes da realização do autocateterismo vesical intermitente - técnica limpa, sendo utilizado água e sabão. Dispensam o uso de qualquer agente antisséptico, luvas ou outros materiais esterilizados.

Gráfico 1 - Distribuição dos pacientes com lesão medular quanto à posição adotada durante a realização do autocateterismo vesical intermitente. Londrina-PR, 1999.

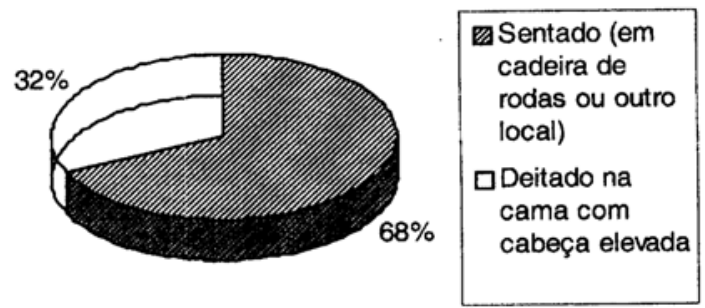

Entre os pacientes homens (20), a maioria, 14 $(70,0 \%)$, realiza o procedimento sentado, $6(30,0 \%)$ porém realizam o autocateterismo deitados. Das duas mulheres que fazem o autocaterismo, uma o faz deitada e a outra sentada.

Froemming(14) indicam para os homens "a realização do cateterismo na posição sentada ou ortostática, introduzindo lentamente o cateter lubrificado na uretra. Se encontrar alguma resistência, recuar o cateter e tentar reintroduzi-lo com movimento giratório e suave. As mulheres também podem realizar o cateterismo na posição sentada ou em pé. Quando sentadas, devem fletir os joelhos e coxas, 
mantendo os pés juntos onde pode ser apoiado um espelho e os joelhos serão afastados um do outro. A paciente também poderá se sentar sobre o vaso sanitário e colocar um espelho sobre um apoio mais baixo".

Lowe; Diokno(16) também concordam que os pacientes do sexo masculino fiquem sentados ao fazerem o autocateterismo e Lelis ${ }^{(12)}$ comenta que o ideal para as mulheres é realizarem o autocateterismo sentadas com os membros inferiores afastados e flexionados.

No nosso estudo, sobre os paraplégicos, notamos, pela experiência dos pacientes, que há necessidade de realizar o primeiro cateterismo do dia deitado, pois a transferência para a cadeira de rodas leva à possível perda urinária.

Gráfico 2 - Distribuição dos pacientes com lesão medular quanto à frequência da realização da higiene intima antes do autocateterismo vesical. LondrinaPR, 1999.
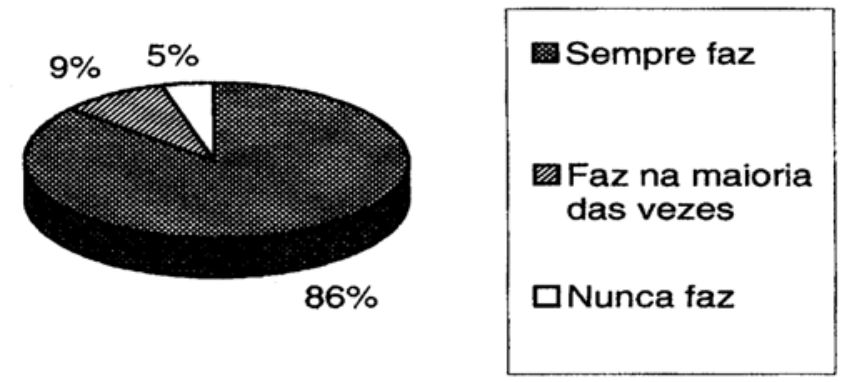

A higiene întima antes da realização do autocateterismo foi uma prática referida pela maioria dos pacientes. A substância mais utilizada foi a água e sabão.

Cerqueira(16) diz que os microorganismos vivem na superficie cutânea, no interior das glândulas sudoriparas, sebáceas e folículos pilosos. Água e sabão, removem facilmente a microbiota exógena.

No períneo e na região inguinal encontra-se Streptococcus faecalis, Proteus mirabilis, Pseudomonas aeruginosas, Escherichia coli e outros.

Gráfico 3 - Distribuição dos pacientes com lesão medular quanto ao uso de lubrificantes para a introdução do cateter. Londrina-PR, 1999.

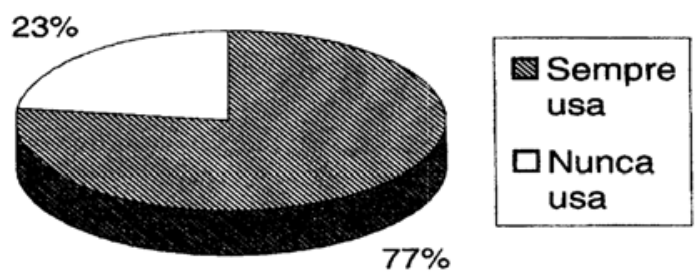

O uso do lubrificante foi uma prática utilizada e referida por $77,0 \%$ dos pacientes, conforme mostra o gráfico 3 . Outros relataram não fazer uso de lubrificante por considerar desnecessário $(23,0 \%)$.

Cerqueira(16), Rodrigues(17) ressaltam a importância do uso de geléia lubrificante estéril e solúvel em água, em cateterismos masculinos a fim de se evitar traumatismo de uretra.

Tabela 2 - Distribuição dos pacientes com lesão medular quanto ao posicionamento do pênis para a introdução do cateter. Londrina-PR, 1999.

\begin{tabular}{lrc}
\hline \multicolumn{1}{c}{ posição } & $\mathbf{n}$ & $\mathbf{\%}$ \\
\hline Faz ângulo reto com o púbis & 16 & 80,0 \\
Lateralizado & 3 & 15,0 \\
Mantém inclinado para baixo & 1 & 5,0 \\
\hline \multicolumn{1}{c}{ TOTAL } & $\mathbf{2 0}$ & $\mathbf{1 0 0 , 0}$ \\
\hline
\end{tabular}

De acordo com os resultados da tabela 2, observou-se que a maioria dos pacientes, 16 (80,0\%), informou posicionar o pênis fazendo ângulo reto com o púbis. Outros $3(15,0 \%)$ informaram que mantêm o pênis lateralizado e um o mantém inclinado para baixo $(5,0 \%)$.

Delisa(18) refere que o curso da uretra masculina é em forma de "S", e que a porção posterior da uretra proximal em homens é circundada pela próstata.

Altshuller(19) referem que a posição do pênis deve ser em ângulo reto com o púbis, esta posição facilita a introdução do cateter pois atenua a curvatura penoescrotal e evita o trauma uretral.

As duas mulheres que responderam a questão referente à exposição da vulva para introdução do cateter, uma delas afirmou que está em orientação há 3 meses, faz uso do espelho e afasta os pequenos e os grandes lábios. A outra paciente informou que não faz uso do espelho, porém mantém afastado.s os pequenos e os grandes lábios para facilitar a introdução do catéter.

Lelis(12) ressalta que o uso de espelho é um importante recurso utilizado em programa de treinamento, uma vez que permite às mulheres o reconhecimento anatômico da região vulvar, com correta identificação do meato uretral, e pode ser dispensado quando a paciente tem segurança $\mathrm{e}$ demonstra familiariedade com esta etapa do procedimento.

Outro recurso indicado por McCaughlin(20), Froemming(14) para identificação do meato uretral é o reconhecimento do local através do tato, utilizando o dedo médio da mão esquerda. 
Tabela 3 - Distribuição dos pacientes com lesão medular quanto aos recipientes utilizados para a coleta de urina drenada na realização do autocateterismo vesical técnica limpa. LondrinaPR, 1999.

\begin{tabular}{lll}
\hline \multicolumn{1}{r}{ recipiente } & n & \multicolumn{1}{c}{ \% } \\
\hline Coletor plástico & 9 & 40,90 \\
Recipiente plástico & 8 & 36,36 \\
Outros recipientes & 2 & 9,09 \\
No vaso sanitário & 1 & 4,55 \\
Cálice graduado & 1 & 4,55 \\
Papagaio & 1 & 4,55 \\
\hline \multicolumn{1}{r}{ TOTAL } & $\mathbf{2 2}$ & $\mathbf{1 0 0 , 0}$ \\
\hline
\end{tabular}

Conforme os resultados, constata-se que o recipiente mais citado para a drenagem de urina foi o coletor plástico $(40,90 \%)$, seguido de recipiente de plástico (bacia, pote de margarina, frasco de álcool vazio), em $36.363 \%$.

Resultados semelhantes também foram encontrados em estudos realizados por Lelis ${ }^{(19)}$ no que diz respeito à drenagem de urina. A maior incidência foi para o uso de recipiente de plástico, papagaio e coletor plástico.

$\mathrm{O}$ uso de tais recipientes não permite mensurar com exatidão o volume drenado, nem caracterizar a cor ou verificar a presença de resíduos, isto prejudica a percepção dos sinais de infecção urinária.

Tabela 4 - Distribuição dos pacientes com lesão medular quanto à mensuração do volume urinário. Londrina-PR, 1999.

\begin{tabular}{lrc}
\hline \multicolumn{1}{c}{ medida do volume urinário } & $\mathrm{n}$ & $\%$ \\
\hline Sempre faz & 10 & 45,5 \\
Faz raramente & 6 & 27,3 \\
Nunca faz & 4 & 18,2 \\
Faz na maioria das vezes & 2 & 9,1 \\
\hline \multicolumn{1}{c}{ TOTAL } & $\mathbf{2 2}$ & $\mathbf{1 0 0 , 0}$ \\
\hline
\end{tabular}

A maioria dos pacientes, $0(45,5)$, informou que procedem à mensuração do volume urinário. Outros, $6(27,3)$, o fazem raramente e alguns nunca o fazem, $4(18,2)$.

Faro(3) ressalta a importância da mensuração do volume urinário drenado, como parâmetro para definir o intervalo das cateterizações. Se o volume residual for maior do que $250 \mathrm{ml}$ fazer cateterismo vesical intermitente a cada 6 horas, e à medida que diminuir o volume drenado o intervalo do cateterismo será aumentado, podendo diminuir o número de cateterismos por dia até torná-lo livre do mesmo.

Lapides $^{(21)}$ relatam a experiência de um paciente que depois de 3 meses de uso do cateterismo intermitente, conseguiu reduzir e manter a urina residual abaixo de $30 \mathrm{ml}$. Considera neste caso que a bexiga foi reabilitada e ressalta a importância da mensuração do volume residual periodicamente.

Tabela 5 - Distribuição dos pacientes com lesão medular segundo a periodicidade, em horas, de realização do autocateterismo vesical intermitente técnica limpa. Londrina-PR, 1999.

\begin{tabular}{|c|c|c|}
\hline periodicidade & $\mathbf{n}$ & $\%$ \\
\hline $3 / 3$ horas & 2 & 9,1 \\
\hline 4/4 horas & 9 & 40,9 \\
\hline $6 / 6$ horas & 8 & 36,4 \\
\hline $8 / 8$ horas & 2 & 9,1 \\
\hline $12 / 12$ horas & 1 & 4,5 \\
\hline TOTAL & 22 & 100,0 \\
\hline
\end{tabular}

Conforme os resultados expressos na tabela 5, observa-se que a maioria dos pacientes (40,9\%) informou realizar o autocateterismo a cada 4 horas, seguido por outros $(36,4 \%)$ que o fazem a cada 6 horas.

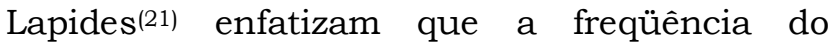
cateterismo é mais importante do que a esterilidade dos materiais, pois o cateterismo a intervalos longos poderá ocasionar a hiperdistensão da bexiga que causa isquemia do detrusor resultando em cistite, pielonefrite e sepsis.

Perkashi(22), relata uma experiência onde inicialmente o intervalo do cateterismo foi de 4 em 4 horas durante as 24 horas até se conseguir micção espontânea, quando então se estendem o intervalo para 6 horas e aumentando-o progressivamente.

Specht(23) e Hunt(24) ressaltam que a freqüência das cateterizações deva ser ajustada de tal forma, que não ultrapasse o volume entre 300 e $400 \mathrm{ml}$ em cada cateterização, pois acima desse valor pode causar hiperdistensão da bexiga levando ao refluxo vesicoureteral.

Segundo Payno(25), uma vez que o paciente começa a urinar espontaneamente entre as cateterizações, devemos medir a urina residual. Quando a urina residual for inferior a $50 \mathrm{ml}$ ou inferior a $10 \%$ da capacidade vesical deve-se suspender as cateterizações.

Hellstrom ${ }^{\mathrm{t}(26)}$, Yokoyama (27) relatam que os pacientes que realizam o autocateterismo com intervalos maiores, entre 8 e 12 horas, e com grande volume de urina pela manhã apresentavam bacteriúria, hidronefrose, deterioração da forma da bexiga e da sua função e algum grau de insuficiência renal.

Os pacientes devem ser instruídos a cateterizarse no tempo previsto, segundo parâmetros clínicos e/ou urodinâmicos (I Consenso Brasileiro de Uroneurologia, 1999). 
Tabela 6 - Distribuição dos pacientes com lesão Mesmo assim, apesar da maioria dos pacientes • 16 medular quanto ao calibre do cateter utilizado no $(73,0 \%)$ reutilizarem o cateter, outros $6(27,0 \%)$ não o autocateterismo vesical intemitente. Londrina-PR, reaproveitam, porque acreditam estar assim 1999.

\begin{tabular}{|c|c|c|}
\hline calibre do catéter & $\mathbf{n}$ & $\%$ \\
\hline Catéter 12 & 16 & 72,73 \\
\hline Catéter 10 & 4 & 18,18 \\
\hline Catéter 14 & 2 & 9,09 \\
\hline TOTAL & 22 & 100,0 \\
\hline
\end{tabular}

prevenindo as infecções urinárias.

De acordo com a tabela, a maioria $16(72,73 \%)$ utiliza o cateter uretral de plástico calibre 12, justificando que este diâmetro facilita a drenagem da urina.

Para Canalini (10) o cateterismo intermitente deve ser feito com cateteres de 6 a $12 \mathrm{Fr}$, selecionando-se o calibre mais apropriado para cada paciente.

Caso, ao introduzir o cateter na uretra, se observar resistência mais acentuada deve-se suspeitar de estreitamento uretral. Nesse caso requer-se uso de cateter menos calibroso e introdução mais cuidadosa(28).

Para maior segurança, temos utilizado nos pacientes que freqüentam o nosso serviço, o cateter $\mathrm{n}^{\circ} 10$ para o primeiro cateterismo e depois passamos para o $\mathrm{n}^{\circ} 12$.

Tabela 7 - Distribuição dos pacientes com lesão medular quanto ao número de vezes que reutilizam o cateter para o autocateterismo vesical intermitente técnica limpa. Londrina-PR, 1999.

\begin{tabular}{lcc}
\multicolumn{1}{c}{ número de vezes } & n & \% \\
\hline até 10 vezes & 7 & 43,75 \\
até 30 vezes & 6 & 37,50 \\
mais de 30 vezes & 3 & 18,75 \\
\hline \multicolumn{1}{c}{ TOTAL } & $\mathbf{1 6}$ & $\mathbf{1 0 0 , 0}$ \\
\hline
\end{tabular}

Obs.: $\mathrm{n}=16$ refere-se ao total de pacientes que reutilizam o cateter; os outros 6 pacientes descartam o cateter após uma utilização.

Quanto ao número de vezes que reutilizam o cateter a maioria dos pacientes $(43,75 \%)$ o fazem até 10 vezes e outros $(37,50 \%)$ até 30 vezes.

$\mathrm{Na}$ literatura há citação de reutilização por 2 semanas e outros por 4 semanas (14,29-30).

Moore (31) não encontrou diferença significante entre os grupos usuários de cateteres esterilizados e os de reaproveitamento do cateter em relação à infecção urinária.

Em 1999, no I Consenso Brasileiro - Incontinência Urinária, Uroneurologia, Disfunções Miccionais, determinou-se que o mesmo cateter pode ser usado por várias semanas, devendo ser trocado mensalmente.

Tabela 8 - Distribuição dos pacientes com lesão medular quanto à substância para a limpeza do cateter para sua reutilização no autocateterismo vesical. Londrina-PR, 1999.

\begin{tabular}{|c|c|c|}
\hline Limpeza do catéter & $\mathbf{n}$ & $\%$ \\
\hline Lava com água e ferve & 8 & 50,0 \\
\hline Lava com água e sabão e ferve & 4 & 25,0 \\
\hline Lava somente com água & 1 & 6,25 \\
\hline Lava com água e sabão & 1 & 6,25 \\
\hline Lava com água e sabão e imerge & 1 & 6,25 \\
\hline em & & \\
\hline Lava com água e imerge em PVPI & 1 & 6,25 \\
\hline TOTAL & 16 & 100,0 \\
\hline
\end{tabular}

Observou-se pelos dados desta tabela diferentes maneiras de reprocessar o cateter, sendo que "lava com água e ferve" e "lava com água e sabão e ferve", obtiveram juntas $75,0 \%$ das respostas.

Karolowicz(30), O'Hagan(32), Froemming(14), Rodrigues ${ }^{(17)}$ recomendam lavar o exterior do cateter com água e sabão e depois enxaguar o seu interior simplesmente com água corrente, secá-lo com toalha limpa e mantê-lo em lugar seco, dentro de um recipiente.

Outros autores, Azevedo(3), Hunt(24) orientam lavar os cateteres com água e sabão, inclusive ferver por 10 minutos.

Wyndaele(29) sugere que se guarde os cateteres em um recipiente em solução aquosa de cloramina 3\% que deve ser trocada semanalmente.

O'Hagan(32) refere que deixar o cateter de molho em solução extremamente asséptica é uma medida desnecessária que deve ser desencorajada, uma vez que isso pode causar irritação da mucosa uretral e destruir a flora normal, abrindo espaço para a colonização secundária.

Esta situação polêmica, foi discutida no I Consenso Brasileiro em Uroneurologia (34) onde definiu-se que o cateter deve ser lavado com água e sabão, enxaguar, secar e guardar em bolsa adequada ou até mesmo embrulhado em papel toalha para o próximo uso. 
Tabela 9 - Distribuição das respostas dos pacientes com lesão medular quanto ao local para o acondicionamento do cateter reutilizado no autocateterismo vesical segundo as respostas dos pacientes. Londrina-PR, 1999.

\begin{tabular}{lrr}
\hline \multicolumn{1}{c}{ local } & n & \multicolumn{1}{c}{ \% } \\
\hline Pano limpo & 5 & 31,25 \\
Marmita de metal & 3 & 18,75 \\
Vidro com tampa de plástico & 2 & 12,5 \\
Coletar plástico de urina & 1 & 6,25 \\
Compressa & 1 & 6,25 \\
Papel da luva & 1 & 6,25 \\
Saboneteira de plástico & 1 & 6,25 \\
Toalha & 1 & 6,25 \\
Vidro com álcool & 1 & 6,25 \\
\hline TOTAL & $\mathbf{1 6}$ & $\mathbf{1 0 0 , 0}$ \\
\hline
\end{tabular}

Diversos materiais foram citados para acondicionar o cateter após a limpeza, desde pano limpo, passado a ferro, marmita de metal e vidro com tampa de plástico.

Outros recipientes foram: coletor plástico, compressa, invólucro de luva, saboneteira, toalha, vidro com álcool.

Podemos perceber que a maioria $(50,0 \%)$ segue as nossas orientações, utilizando pano limpo e marmita de metal, que oferecem segurança na manutenção da limpeza e evitam deformidade do cateter.

Outros aspectos que envolvem o procedimento eque merecem novos estudos são: profissionais que orientaram o procedimento, em qual momento foram orientados, há quanto tempo realizam o procedimento equais os locais utilizados na execução do autocateterismo, divulgando e contextualizando tal procedimento.

\section{CONCLUSÃo}

- A maioria dos pacientes entrevistados $(68,0 \%)$ informou realizar o procedimento sentado.

- A higiene das mãos é feita em sua maioria com água e sabão antes da introdução do cateter $(86,4 \%)$

e após o procedimento $(81,82 \%)$, sendo que $71,44 \%$ usa a mesma substância para higiene íntima.

- O uso de geléia lubrificante foi relatado pela maioria dos entrevistados $(77,0 \%)$.

- O posicionamento do pênis fazendo ângulo reto com o púbis para a introdução do cateter é referido pela maioria dos pacientes $(80 \%)$.

- Ambas as mulheres pesquisadas mantinham afastados os pequenos e grandes lábios para a introdução do cateter.
- A maioria não utiliza luvas tanto para introduzir quanto para retirar o cateter.

- O recipiente para a drenagem de urina mais citado foi o coletor plástico $(40,30 \%)$. Podemos ressaltar que diferentes recipientes plásticos, tais como potes de margarina, de maionese, também foram utilizados de acordo com a criatividade e a disponibilidade do paciente.

- Todos 100\% dos pacientes afirmavam que retiravam o cateter com a finalização da drenagem urinária.

- A mensuração do volume urinário é feita pela maioria dos pacientes entrevistados $(54,6 \%)$.

- O intervalo entre os procedimentos variaram de $3 \mathrm{em} 3$ horas até de $12 \mathrm{em} 12$ horas com predominância de $4 \mathrm{em}$ 4 horas $(40,9 \%)$, sendo o cateter de calibre 12 o mais utilizado $(72,73 \%)$ por esta população.

- A maioria dos pacientes pesquisados $(73,0 \%)$ reutiliza o cateter variando a reutilização entre 2 e 120 vezes, sendo que $81,25 \%$ o reutiliza até 30 vezes.

- A limpeza do cateter é feita predominantemente com água. Muitos $(75,0 \%)$ complementam a limpeza fervendo-o ou imergindo em água quente. Após a limpeza os cateteres são acondicionados de maneiras variadas, predominando o uso de pano limpo $(31,25 \%)$.

\section{CONSIDERAÇÕES FINAIS}

Pretendemos, com este estudo, descrever a realização do autocateterismo vesical interminte, em domicílio, visando constatar a assimilação das orientações recebidas e o modo como estes pacientes com lesão medular vêm estruturando o seu procedimento.

A medida que o paciente evolui para um quadro estável, deve-se orientá-lo quanto ao seu estado fisiológico atual, seu novo corpo, conscientizando-o de sua responsabilidade pela reabilitação. Devem-se fornecer essas orientações de maneira positiva e otimista, de modo a infundir nele o desejo de cooperar na melhora de suas atividades diárias, já durante a internação hospitalar.

Dentre os inúmeros cuidados ao paciente com lesão medular, podemos ressaltar a necessidade do autocateterismo vesical intermitente técnica limpa, que é um procedimento com vários beneficios, tornando o paciente independente e preservando o sistema urinário.

Este procedimento, quando bem orientado, proporciona ao paciente com lesão medular, a oportunidade de convivência social, levando-o ao retorno de suas atividades profissionais, culturais e de lazer.

Quanto ao enfermeiro, no que tange às suas perspectivas profissionais, é urgente que compreenda o indivíduo com lesão medular no contexto da família, 
como alguém produtivo que pode, através de um atendimento domiciliário estruturado, manter-se em casa, com possibilidade de levar vida normal, ficando o hospital com mais vagas liberadas.

Os pacientes, quando bem orientados, levam uma vida normal, sem infecção e sem complicações secundárias a lesão medular.

\section{REFERÊNCIAS BIBLIOGRÁFICAS}

(1) Demenech AA. Lesado medular - uma ajuda. Enfoque 1989; 17 (1): 19-25.

(2) Lopes MB. Paciente lesado medular: estudo de alguns fatores que interferem na assistência de enfermagem [monografia]. Londrina: Universidade Estadual de Londrina; 1988.

(3) Faro ACM. O ensino de reabilitação: expectativas de estudantes de enfermagem. Rev Esc Enferm USP 1996; 30 (2): 332-9.

(4) Faro ACM. Do diagnóstico à conduta de enfermagem: a trajetória do cuidar na reabilitação do lesado medular [tese]. São Paulo: Escola de Enfermagem de Ribeirão Preto/USP; 1998.

(5) Lianza S. Órtese de propulsão recíproca modelo Argo método de avaliação, tratamento e análise de resultados na reeducação da locomoção em paciente s com lesão medular [tese]. São Paulo: Faculdade de Ciências Médicas; 1997.

(6) Faro ACM. A disfunção sexual enquanto diagnóstico de enfermagem - características definidoras encontradas em lesados medulares. Rev Esc Enferm USP 1995; 29 (2): 166-72.

(7) Guttmann L. Spinal cord injuries: comprehensive management and research. Oxford: Blackwell Scientific Publications; 1973.

(8) Lapides J, Diokno AC, Silber SM, Lowe BS. Clean, intermittent self-catheterization in the treatment of urinary tract disease. J Urol 1972; 107: 458-61.

(9) Lianza S (coordenador). Medicina de reabilitação. 2.ed. Rio de Janeiro: Guanabara Koogan; 1995. A lesão medular; p.304-26.

(10) Canalini AF, Otton RA, Rubinstein I, Aguinaga S. Bexiga de choque. Med HUPE - UERJ 1989; 8 (2): 135-43.

(1 1) Illanes RG. Experiência con cateterismo intermitente. Rev Chilena Urologia 1986; 49: 41-4.

(12) Lelis MAS. Cateterismo vesical intermitente - técnica limpa: caracterização da prática vivenciada por um grupo de pacientes [dissertação]. São Paulo: Escola de Enfermagem da Universidade de São Paulo; 1998.

(13) Kawagoe JY, Queiroz ML. Normas Básicas para a realização de procedimentos. In: Rodrigues EAC, Mendonça JSM, Amarante JMB. Infecções hospitalares: prevenção e controle. São Paulo: Sarvier; 1997. p.58-66.

(14)Froemming C, Smaniotto ML, Lima CLM. Cateterismo vesical intermitente. Rev HCPA. 1988, 8 (1): 29-35.

(15) Lowe BS, Diokno AC. Intermittent self-catheterization. In: Broadwell DC, Jackson BJ. Principles of ostomy care. St Louis: Mosby; 1982. p.770-82.
(16) Cerqueira MCM. Antissepia: principios gerais e antissépticos. In: Rodrigues EAC, Mendonça JSM, Amarente JMB. Infecções hospitalares: prevenção e controle. São Paulo: Sarvier; 1997. p.426-438.

(17) Rodrigues EAC. Infecções do trato urinário. In: Rodrigues EAC, Mendonça JS, Amarante JMB. Infecções hospitalares: prevenção e controle. São Paulo: Sarvier; 1997. p. 135-48.

(18) Delisa JA. Medicina de reabilitação: princípios e prática. São Paulo: Manole; 1992.

(19) Altshuller A, Meyer J, Butz MKJ. Even children can learn to do clean self-catheterization. Am J Nurs 1977; 77 (1): 97101 .

(20) McLaughlin JF. Practical procedures. Dev Med Child Neurol 1996; 38: 446-54.

(2 1) Lapides J, Diokno AC, Lowe BS, Kalish MD. Followup on unsterile, intermittent self-catheterization. J Urol 1974; 111: $184-7$.

(22) Perkash I. Intermittent catheterization and bladder rehabilitation in spinal cord injury patients. $J$ Urol 1975; 114: 230-3.

(23) Specht JP, Maas ML, Wille TTS, Myers NK. Intermittent catheterization. In: Bulechek GM, McCloskey JC. Nursing Interventions. 2.ed. Philadelphia: W.B. Saunders; 1992. p.61-72.

(24) Hunt GM, Oakeshott P, Whitaker RH. Intermittent catheterisation: simple, safe and effective but underused. BMJ 1996; 312 (13): 103-7.

(25) Payno PB. Nuestra experiencia en las infecciones urinarias en los paraplejicos. Rehabilitacion 1981; 15(2): 235-42.

(26) Hellstrõm P, Tammela T, Lukkarinen O, Kontturi M. Efficacy and safety of clean intermittent catheterization in adults. Eur Urol 1991; 20 (2): 117-21.

(27) Yokoyama O, Hasegawa T, Ishiura Y, Ohkawa M, Sugiyama Y, Izumida S. Morphological and functional factors predicting bladder deterioration after spinal cord injury. J Urol 1996; 155: 271-4.

(28) Prado AR, Dantas LS. Cateterismo vesical. JBM 1989; 57(1): 25-30.

(29) Wyndaele $J J$, Oosterlinck W, De Sy W. Clean intermittent self-catheterization in the chronical management of the neurogenic bladder. Eur Urol 1980; 6:107-10.

(30) Karolowicz KA, Meredith CE. Adult voiding dysfunction. In: Karolowicz KA. Urologic nursing, principles and pratice. Philadelphia: W.B. Saunders; 1995. p.377-407.

(3 1) Moore KN. Intermittent self-catheterization: research-based practice. Br J Nurs 1995; 4(18): 1057-63.

(32) O'Hagan M. Neurogenic bladder dysfunction. In: Jones R, Agnes. Nursing for continence. 2.ed. England: HODH; 1996.

(33) Azevedo MAJ, Maria, MLSS, Soler, CMA. Promovendo o auto-cuidado - treinamento e assistência de enfermagem a pacientes portadores de bexiga neurogênica. Rev Bras Enferm 1990; 43 (1, 2, 3/4): 52-7.

(34) I Consenso Brasileiro - Incontinência urinária, Uroneurologia, Disfunções Miccionais. São Paulo; 1999.

Artigo recebido em 25/06/02 Artigo aprovado em 17/03/03 